\title{
Vorzüge und Schattenseiten der Flexibilität der Dauer der Arbeitszeiten. Eine Untersuchung in sieben österreichischen Branchen
}

\author{
Bettina STADLER ${ }^{1}$ \\ Forschungs- und Beratungsstelle Arbeitswelt Wien
}

\begin{abstract}
Mit dem Beitrag wird eine Zuordnung verschiedener Formen der Arbeitszeitgestaltung zu vorwiegend arbeitnehmerInnen- und arbeitgeberInnenseitiger Flexibilisierung vorgenommen und die Auswirkungen dieser flexiblen Arbeitszeiten auf bezahlte und unbezahlte Mehrarbeitsstunden untersucht. Weiter haben wir gefragt, unter welchen Bedingungen flexible Arbeitszeiten von den ArbeitnehmerInnen positiv und in welchen Fällen diese negativ bewertet werden. Basis für die Analyse ist eine Befragung österreichischer ArbeitnehmerInnen in insgesamt sieben Branchen.

Die Untersuchung zeigt, dass flexible Arbeitszeiten häufiger mit Mehrarbeit verbunden sind als insgesamt wenig flexible Arbeitszeiten. Zusätzlich leisten höher Gebildete häufiger Mehrarbeit. Wird am Abend oder am Samstag gearbeitet, ist dies ebenfalls häufig mit Mehrarbeit verbunden. Von diesen Zusammenhängen unterscheiden sich die Risikofaktoren für unbezahlte Mehrarbeit deutlich. Hier treten vor allem zwei Branchen, die Gastronomie und der Bankensektor, hervor. Zusätzlich haben geringer Gebildete ein signifikant höheres Risiko auf unbezahlte Mehrarbeit. Trotz des vorhandenen Risikos für Mehrarbeit wird Flexibilisierung häufig positiv bewertet. Dies ist vor allem der Fall, wenn die ArbeitnehmerInnen in die Gestaltung flexibler Arbeitszeiten eingebunden sind. Sind die Arbeitszeiten dagegen vorwiegend nach den Anforderungen der ArbeitgeberInnen bzw. den Arbeitsanforderungen geformt, führt dies zu negativeren Bewertungen des Arbeitszeitmodells.
\end{abstract}

\section{Einführung}

Bereits seit mehreren Jahrzehnten wird in den Sozialwissenschaften eine intensive Debatte über flexible Arbeitszeiten, ihre Vorzüge und Schattenseiten geführt (für einen Überblick: Seifert 2005). Beobachtet wird zum einen, dass flexiblere Arbeitszeiten die Vereinbarkeit von Familie und Beruf verbessern (Jürgens 2005; Lott 2014; Skinner/Pocock 2011; Wunder/Heineck 2013). Zum anderen wurde wiederholt gezeigt, dass flexible Arbeitszeiten zu langen und manchmal unvollständig abgegoltenen Arbeitszeiten führen können (exemplarisch: Saupe/Stadler 2016). Hinzu kommt, dass durch neue technologische Möglichkeiten die Gren-

\footnotetext{
${ }^{1}$ Bettina Stadler ist wissenschaftliche Mitarbeiterin bei der Forschungs- und Beratungsstelle Arbeitswelt, Wien (FORBA) und lehrt Methoden der empirischen Sozialforschung an der Universität Wien: stadler@forba.at.
}

DOI: http://dx.doi.org/10.18753/2297-8224-109 
zen zwischen Arbeit und Nicht-Arbeit in vielen Bereich immer mehr verschwimmen (Flecker 2017; Jürgens 2005; Moldaschl/Voß 2003; Voß 1998). Zusätzlich wird beobachtet, dass Arbeit durch Flexibilisierung intensiviert wird. Von Unternehmensseite kann durch flexible Anpassung der Arbeitszeiten an den tatsächlichen Bedarf des Unternehmens die Produktivität der ArbeitnehmerInnen erhöht werden (Flecker 2017; Herrmann et al. 2015) . Steh- oder Leerzeiten von ArbeitnehmerInnen sollen so der Vergangenheit angehören. Diese zum Teil sehr intensiv geführte Flexibilisierungsdebatte hat in den letzten Jahren den früher dominierenden Diskurs zum Thema Arbeitszeitverkürzung in den Hintergrund treten lassen, obwohl vor allem von Gewerkschaftsseite weiterhin Forderungen in diese Richtung erhoben werden (Astleithner/Flecker 2017: 23; Flecker \& Altreiter 2014; Reiner 2015).

Mit diesem Artikel sollen die Formen und Auswirkungen flexibler Arbeitszeiten auf Basis empirischer Evidenz für Österreich näher untersucht werden und so ein Beitrag zur Diskussion über Formen, Chancen und Risiken flexibler Arbeitszeiten geleistet werden. ${ }^{2}$

\section{Theoretischer Rahmen}

Flexible und insbesondere selbstgesteuerte Arbeitszeiten versprechen eine deutliche Erhöhung der Lebensqualität von ArbeitnehmerInnen (Eichmann/Saupe 2014). So können ArbeitnehmerInnen - in einem Rahmen betrieblicher Erfordernisse und eventuell vorhandener zeitlicher Rahmenvorgaben - mit flexiblen Arbeitszeiten selbst entscheiden, wann sie arbeiten und wann sie ihre Zeit für andere Vorhaben, seien es Betreuungsaufgaben, soziales Engagement oder individuelle Freizeitaktivtäten, verwenden (Skinner/Pocock 2011). Arbeitsleistung wird in einem Teil dieser Kontexte nicht mehr in geleisteten Stunden, sondern in erreichten Zielvorgaben gemessen (kritisch hierzu: Peters \& Sauer 2005). In der Praxis führen flexible und selbstgesteuerte Arbeitszeiten jedoch häufig zu überlangen Arbeitszeiten. ArbeitnehmerInnen scheint es in vielen Fällen schwer zu fallen, vor der körperlichen und/oder physischen Erschöpfung selbst Grenzen bei ihrer Arbeitszeit einzuziehen (Matta 2015). Besonders gilt dies für Vollzeiterwerbstätige und Menschen ohne die Arbeitszeit limitierende Betreuungsverantwortung (Lott 2014). In qualitativen Interviews wird von ArbeitnehmerInnen dazu auch manchmal berichtet, dass die zugeteilte Arbeitsmenge keine anderen Optionen offen lassen als immer wieder über das Maß der traditionellen Normalarbeitszeit hinaus zu arbeiten (Schönauer et al. 2016).

Autoren wie Klaus Dörre sehen durch eine zunehmende Aufhebung der traditionellen zeitlichen Grenzen zwischen Arbeit und Leben eine weitere „Landnahme“ kapitalistischer Produktionsverhältnisse. „Die Durchsetzung flexibler Produktionsweisen geht mit einer weit reichenden Beschneidung der Zeitsouveränität gesellschaftlicher Majoritäten einher." (Dörre 2010: 66).

Zugleich werden flexible Arbeitszeiten - das zeigen auch die hier präsentierten Ergebnisse- von vielen ArbeitnehmerInnen wegen der gebotenen Möglichkeiten der Gestaltung geschätzt (Lott 2014). Insbesondere gilt dies für Menschen, darunter in erster Linie Frauen, mit Betreuungsverantwortung. Gerade für diese Gruppe erleichtern flexible Arbeitszeiten die Er-

\footnotetext{
${ }^{2}$ Die Autorin dankt zwei nicht namentlich genannten GutachterInnen für wertvolle Hinweise.
} 
füllung von unterschiedlichsten und nur zum Teil schon im Voraus planbaren Zeiterfordernissen. Zugleich wird durch die häufig unaufschiebbare Natur von Betreuungspflichten auch die Verfügbarkeit von ArbeitnehmerInnen für berufliche Belange eingeschränkt und arbeitgeberInnenseitige Flexibilisierung stößt bei dieser Gruppe an Grenzen, mit möglicherweise negativen Auswirkungen auf Karriere und Gehalt.

Um differenziertere Analysen der Auswirkungen flexibler Arbeitszeiten vornehmen zu können, wird in manchen Forschungsarbeiten zwischen arbeitnehmerInnenseitig gesteuerten flexiblen Arbeitszeiten und arbeitgeberInnenseitig gesteuerten Arbeitszeiten unterschieden (Lott 2015, Griesbacher/Griesbacher 2016). Dabei können diese beiden Formen sowohl als einander entgegengesetzt gedacht werden als auch als unterschiedliche Dimensionen, die gleichzeitig in verschiedenen Konstellationen auftreten können (für eine Überblick: Chung \& Tijdens 2013). Für die hier präsentierten Analysen wird davon ausgegangen, dass flexible Arbeitszeitarrangements sowohl von Interessen der ArbeitnehmerInnen als auch der ArbeitgeberInnen motiviert sind. In der realen Ausgestaltung im Arbeitsalltag kann jedoch von einer Dominanz der Interessen der ArbeitgeberInnen oder der Interessen der ArbeitnehmerInnen ausgegangen werden.

Mit diesem Beitrag werden die folgenden Fragen untersucht und so ein Beitrag zur Diskussion der Formen und Wirkungen flexibler Arbeitszeiten geleistet: Lässt sich empirisch zeigen, dass flexible Arbeitszeiten in Österreich zu längeren Arbeitszeiten und in weiterer Folge vermehrt zu unbezahlten Über- und Mehrstunden führen? Gibt es hierbei Unterschiede zwischen arbeitnehmerInnen- und arbeitgeberInnenseitig motivierter Flexibilität? Wie wirken diese Formen flexibler Arbeitszeiten, ist ein Zusammenhang zwischen den Formen flexibler Arbeitszeitgestaltung und der Zufriedenheit von ArbeitnehmerInnen mit ihrem Arbeitszeitmodell erkennbar?

\section{Daten und Methode}

Basis für die Analyse ist eine Befragung von ArbeitnehmerInnen in Österreich im Jahr 2015. Dabei handelt es sich um eine einmalig durchgeführte Befragung. Die Stichprobe der Befragung wurde aus den Mitgliederverzeichnissen der österreichischen Arbeiterkammern gezogen. In Österreich sind, mit wenigen Ausnahmen, ${ }^{3}$ alle unselbständig Erwerbstätigen verpflichtend Mitglied bei einer Interessenvertretung - den Arbeiterkammern. ${ }^{4}$ Aus dem Mitgliederverzeichnis dieser Einrichtungen wurde eine Stichprobe von unselbständig Beschäftigten in sieben Branchen als Zufallsstichprobe gezogen.

Für die Befragung wurde von einer Gruppe von ArbeitsrechtsexpertInnen und ForscherInnen ein eigener Fragebogen entwickelt. Zentrales Ziel der Befragung war, die Perspektive österreichischer ArbeitnehmerInnen auf flexible Arbeitszeiten detailliert zu erfassen. Besonderes Interesse galt den Mustern der Gestaltung der Arbeitszeit, der Abgeltung von Mehrarbeitsstunden und der Zufriedenheit mit den verschiedenen Arbeitszeitmodellen. Mit der Be-

\footnotetext{
${ }^{3}$ Nicht von der Pflichtmitgliedschaft erfasst sind u.A. Führungskräfte der ersten Führungsebene und GeschäftsführerInnen von Kapitalgesellschaften, LehrerInnen und einige weitere kleine Gruppen am Arbeitsmarkt.

${ }^{4}$ Dieses Organ der Interessenvertretung ist föderal organisiert, d.h. es gibt in jedem der neun österreichischen Bundesländer eine eigene Arbeiterkammer.
} 
fragung wurden die verschiedenen in Österreich gesetzlich vorgesehenen und praktisch vorhandenen Arbeitszeitmodelle abgedeckt. Konkret wurden die folgenden Modelle erfragt: Gleitzeit, Schichtarbeit, jede Woche gleiche Arbeitsstunden und schwankende Arbeitszeiten. Eine detaillierte Beschreibung dieser Modelle und ihrer Abgrenzung folgt im nächsten Abschnitt.

Insgesamt wurden 2008 ArbeitnehmerInnen telefonisch befragt. Die Befragung erfasste die Branchen Metall (Industrie und Gewerbe), Elektroindustrie, Gastronomie und Hotellerie, IT, Banken, Reinigung und Handel. Damit sollte in Hinblick auf die Qualifikation der ArbeitnehmerInnen, der Dominanz von weiblichen und männlichen Beschäftigten und den vorherrschenden Arbeitszeitmodellen ein breites Spektrum an Branchen abgedeckt werden. Mit der Entscheidung der Auswahl von Branchen sind die hier präsentierten Ergebnisse zwar repräsentativ für die ausgewählten Branchen, nicht jedoch für die unselbständig Erwerbstätigen in Österreich insgesamt. Die Anzahl der Befragten in den verschiedenen Branchen wird in Tabelle 1 wiedergegeben.

Tabelle 1: Anzahl der Befragten nach Branchen

\begin{tabular}{|l|l|l|}
\hline Branchen & \multicolumn{2}{|l|}{} \\
\hline & absolut & Spalten\% \\
\hline Gesamt & 2008 & 100 \\
\hline Metall (Industrie oder Gewerbe) & 398 & 19,8 \\
\hline Elektroindustrie & 267 & 13,3 \\
\hline Gastronomie und Beherbergung & 277 & 13,8 \\
\hline IT & 255 & 12,7 \\
\hline Banken & 272 & 13,5 \\
\hline Reinigung & 274 & 13,6 \\
\hline
\end{tabular}

Quelle: FORBA-Erhebung zu Arbeitszeitflexibilisierung 2015

Nach einem Überblick über die Verbreitung der verschiedenen Modelle der Arbeitszeitgestaltung wird näher auf ihre Bedeutung in den sieben untersuchten Branchen eingegangen. Anschließend wird die Operationalisierung des Konzeptes arbeitnehmerInnen- und arbeitgeberInnenseitiger Flexibilität vorgestellt und mit Hilfe von zwei binären logistischen Regressionsmodellen die Wirkung dieser Formen der Flexibilisierung auf Mehrarbeit und unbezahlte Überstunden untersucht. Diese Faktoren werden in den Kontext der erfassten Branchen, der unterschiedlichen Arbeitszeitmodelle, der Lage der Arbeitszeit und weiterer Faktoren gestellt. Abschließend wird dem Zusammenhang von Arbeitszeitmodell und der Form der Arbeitszeitflexibilisierung mit der Frage nach der Zufriedenheit mit dem Arbeitszeitmodell und der Zufriedenheit mit der Vereinbarkeit von Arbeit und außerberuflichen Verpflichtungen nachgegangen. ${ }^{5}$

\footnotetext{
${ }^{5}$ Die Zufriedenheit mit dem Arbeitszeitmodell wurde auf einer vierstufigen Skala erfasst.
} 


\section{Muster der Flexibilisierung in verschiedenen Arbeitszeitmodellen}

Für die hier durchgeführte Analyse ist eine Vergröberung der gesetzlich definierten und praktisch auffindbaren Formen der Gestaltung der Arbeitszeit notwendig. Im Folgenden wird zwischen den folgenden Arbeitszeitmodellen unterschieden:

- Jede Woche die gleiche Arbeitszeit: Hier ist ein Normalarbeitszeitmodell angesprochen, das außer Über- oder Mehrstunden keine Abweichungen von der vertraglich vereinbarten Wochenarbeitszeit vorsieht. In der klassischen Variante bleibt auch die Lage der Arbeitszeit von Woche zu Woche gleich; es gibt allerdings auch die Möglichkeit einer wechselnden Verteilung der gleichbleibenden Stundenanzahl auf die Arbeitswoche. Beide Ausprägungen, die Arbeit zu immer gleichen Zeiten als auch gleiche Arbeitszeiten in wechselnder Lage, wurden mit dieser Kategorie erfasst.

- Gleitzeit: Im österreichischen Arbeitszeitgesetz findet sich die Möglichkeit einer betrieblichen Gleitzeitvereinbarung, die die Beschäftigten ermächtigt, Beginn und Ende ihrer täglichen Arbeitszeit innerhalb eines betrieblich definierten Rahmens selbst zu bestimmen. Dabei lässt die gesetzliche Regelung den betrieblichen Vereinbarungen viel Spielraum. Die Gleitzeitvereinbarung muss den Gleitzeitrahmen und die sogenannte Gleitzeitperiode, d.h. den Durchrechnungszeitraum, festlegen, außerdem das Ausmaß, in dem Zeitguthaben oder -schulden aus einer Gleitzeitperiode in die nächste mitgenommen werden können. Für die Länge der Gleitzeitperiode und die Mitnahme von Zeitguthaben oder -schulden werden keine Höchstgrenzen festgelegt. Das Arbeitszeitgesetz erlaubt damit recht flexible Ausgestaltungen des definierten Rahmens für Gleitzeitmodelle. Zur Nutzung dieser Möglichkeiten durch die österreichischen Betriebe liegen wenig empirische Daten vor. Dies gilt auch für die Frage, inwieweit die der arbeitsrechtlichen Gleitzeitregelung zugrundeliegende Intention, die Arbeitszeitautonomie der Beschäftigten zu erhöhen, in der praktischen Umsetzung von Gleitzeitmodellen auffindbar ist, bzw. in welchem Ausmaß Gleitzeitmodelle auch im Interesse der ArbeitgeberInnen eingesetzt werden, insbesondere zur Einsparung von Mehrarbeitszuschlägen.

- Schichtarbeit: diese Form der Arbeitszeitgestaltung liegt nach der hier verwendeten Definition vor, wenn ein Arbeitsplatz an einem Arbeitstag von mehreren einander abwechselnden (Gruppen von) ArbeitnehmerInnen eingenommen wird bzw. wenn Arbeitsgruppen in bestimmten Betriebsabteilungen einander zeitlich nachfolgend ablösen. Hier ist sowohl Schichtarbeit in der Industrie mit ausgearbeiteten Schichtplänen als auch die Organisation der Arbeit in wechselnden Schichten im Dienstleistungssektor wie beispielsweise im Gastgewerbe, angesprochen. Diese Schichtmodelle umfassen häufig Arbeit an den Rändern des Tages, am Abend, in der Nacht oder am Wochenende (vgl. Darstellung in Tabelle 2).

- Schwankende Arbeitszeit, aber weder Schicht noch Gleitzeit: Hier handelt es sich um eine Art Residualkategorie. Einerseits fallen hierunter Beschäftigte, die in einem Durchrechnungs- bzw. Bandbreitenmodell arbeiten, d.h. ihre Normalarbeitszeit kann in Phasen erhöhten Arbeitsanfalls arbeitgeberInnenseitig erhöht werden, anschließend wird diese Mehrarbeit wieder ausgeglichen, sodass die vertraglich vereinbarte Normal- 
arbeitszeit über den festgelegten Durchrechnungszeitraum im Durchschnitt nicht überschritten wird. In diese Kategorie fallen aber auch Beschäftigte, deren Arbeitszeit in einer Weise schwankt, die von arbeitsrechtlichen Mindeststandards abweicht. Beispiele dafür bilden Angestellte in Gastronomie oder Handel, die nach sehr kurzfristig bekanntgegebenen, wöchentlich wechselnden Dienstplänen arbeiten, wobei die gesetzliche Ankündigungsfrist von (normalerweise) vierzehn Tagen immer wieder unterschritten wird.

Wie Tabelle 1 zeigt, sind diese Modelle der Arbeitszeitgestaltung in den sieben untersuchten Branchen unterschiedlich stark vertreten.

Tabelle 1: Modelle der Arbeitszeitgestaltung in den Branchen

\begin{tabular}{|c|c|c|c|c|c|}
\hline & $\begin{array}{l}\text { Jede Woche } \\
\text { gleiche Stun- } \\
\text { den }\end{array}$ & $\begin{array}{l}\text { Mit schwan- } \\
\text { kender Stun- } \\
\text { denanzahl }\end{array}$ & $\begin{array}{l}\text { In einem } \\
\text { Gleitzeit- } \\
\text { modell }\end{array}$ & $\begin{array}{l}\text { In regel- } \\
\text { mäßigen } \\
\text { Schichten }\end{array}$ & Gesamt \\
\hline & \multicolumn{5}{|c|}{ Zeilen\% } \\
\hline Gesamt & $40 \%$ & $14 \%$ & $31 \%$ & $15 \%$ & $100 \%$ \\
\hline Banken & $50 \%$ & $6 \%$ & $42 \%$ & $2 \%$ & $100 \%$ \\
\hline Elektroindustrie & $24 \%$ & $5 \%$ & $42 \%$ & $28 \%$ & $100 \%$ \\
\hline Gastronomie & $49 \%$ & $30 \%$ & $5 \%$ & $15 \%$ & $100 \%$ \\
\hline Handel & $50 \%$ & $20 \%$ & $17 \%$ & $13 \%$ & $100 \%$ \\
\hline IT & $15 \%$ & $8 \%$ & $74 \%$ & $3 \%$ & $100 \%$ \\
\hline Metall & $33 \%$ & $6 \%$ & $32 \%$ & $29 \%$ & $100 \%$ \\
\hline Reinigung & $56 \%$ & $23 \%$ & $10 \%$ & $11 \%$ & $100 \%$ \\
\hline
\end{tabular}

Quelle: FORBA-Erhebung zu Arbeitszeitflexibilisierung 2015.

Der im Gesamtdurchschnitt größte Anteil der ArbeitnehmerInnen (40\%) aus den sieben untersuchten Branchen hat mit den ArbeitgeberInnen eine wöchentlich gleichbleibende Anzahl an Arbeitsstunden vereinbart. Diese fixen Arbeitsstunden können entweder auch zu den immer gleichen Zeiten geleistet werden oder die Verteilung der Arbeitszeit variiert von Woche zu Woche. In der Reinigung arbeiten 56\% der ArbeitnehmerInnen in diesem Modell, im Bereich der Banken und des Handels ist dies jeweils genau die Hälfte der Beschäftigten. Selten sind wöchentlich gleichbleibende Arbeitszeiten in der IT (15\%) und der Elektroindustrie (24\%).

Gleitzeitmodelle sind unter allen untersuchten Branchen in der IT am meisten verbreitet, hier arbeiten 74\% der Beschäftigten in einem solchen Modell. An zweiter Stelle folgen der Bankensektor und die Elektroindustrie. Jeweils 42\% der ArbeitnehmerInnen im Bankensektor und in der Elektroindustrie arbeiten in Gleitzeit. Im Metallsektor sind dies 32\%. In den direkt an KundenInnenaufkommen und KundenInnenwünschen ausgerichteten Branchen Gastgewerbe (5\%), Reinigung (10\%) und Handel (17\%) kommt nur wenig Gleitzeit vor.

In regelmäßigen Schichten arbeiten insgesamt 15\% der Befragten, deutlich höher ist dieser Anteil in der Metall- und der Elektroindustrie mit 29\% bzw. 28\% der Beschäftigten. Aber auch 
in der Gastronomie ordnen sich 15\% und im Handel 13\% der Beschäftigten dem Modell regelmäßig wiederkehrender Schichten zu. Mit wöchentlich schwankender Stundenanzahl aber ohne Gleitzeit- oder Schichtmodell arbeiten schließlich insgesamt 14\% der Befragten. Von größerer Bedeutung ist dieses Modell in der Gastronomie (30\%) und in der Reinigung (23\%).

Die vier gewählten Modelle der Arbeitszeit grenzen sich nicht nach Lage der Arbeitszeit ab. Aus diesem Grund wird in Tabelle 2 dargestellt, wie häufig von den verschiedenen Gruppen Arbeit am Abend, in der Nacht oder am Wochenende geleistet wird. Für die Darstellung wurden die Antwortmöglichkeiten „Mehrmals pro Woche“ und „Mehrmals im Monat“ zur Kategorie „Mehrmals im Monat/jede Woche“ zusammengefasst.

Tabelle 2: Lage der Arbeitszeit in den Arbeitszeitmodellen

\begin{tabular}{|l|c|c|c|c|}
\hline & $\begin{array}{l}\text { Arbeit am } \\
\text { Abend }\end{array}$ & $\begin{array}{l}\text { Arbeit in der } \\
\text { Nacht }\end{array}$ & $\begin{array}{l}\text { Arbeit am } \\
\text { Samstag }^{6}\end{array}$ & $\begin{array}{l}\text { Arbeit am } \\
\text { Sonntag }^{6}\end{array}$ \\
\hline Gesamt & \multicolumn{2}{|c|}{ Anteil „Mehrmals im Monat/in der Woche“ } \\
\hline Jede Woche gleiche Stunden? & $19 \%$ & $9 \%$ & $22 \%$ & $13 \%$ \\
\hline Mit schwankender Stundenanzahl & $10 \%$ & $5 \%$ & $22 \%$ & $11 \%$ \\
\hline In einem Gleitzeitmodell & $27 \%$ & $12 \%$ & $42 \%$ & $26 \%$ \\
\hline In regelmäßigen Schichten & $9 \%$ & $3 \%$ & $6 \%$ & $3 \%$ \\
\hline
\end{tabular}

Quelle: FORBA-Erhebung zu Arbeitszeitflexibilisierung 2015.

Insgesamt arbeiten 19\% der ArbeitnehmerInnen in den untersuchten sieben Branchen immer wieder am Abend, noch deutlich höher liegt dieser Anteil bei den Beschäftigten in Schichtarbeit, dort arbeitet etwas mehr als Hälfte der Beschäftigten regelmäßig am Abend, und bei Beschäftigten mit schwankender Stundenzahl. Während der Nacht arbeiten 9\% der Interviewten. Ebenfalls sehr viel stärker von Nachtarbeit sind Beschäftigte mit Schichtarbeit betroffen. Mindestens mehrmals im Monat am Samstag arbeiten vier von zehn ArbeitnehmerInnen mit schwankender Stundenanzahl, gefolgt wiederum von Beschäftigten in regelmäßigen Schichten. Vermehrte Arbeit am Sonntag wird ebenfalls von einem Viertel der Beschäftigten mit schwankenden Arbeitszeiten und jenen mit Schichtdiensten geleistet.

\section{ArbeitgeberInnenseitige vs. Arbeitnehmerlnnenseitig bestimmte Flexibilisierung}

Das Ausmaß, in dem Flexibilität innerhalb eines Arbeitszeitmodells von den ArbeitgeberInnen bzw. von den ArbeitnehmerInnen bestimmt wird, bildet einen wichtigen Bezugspunkt für die Bewertung unterschiedlich flexibler Arbeitszeitarrangements. In Abgrenzung zu Chung und Tidjens (2013) wird hier von dominierenden Interessen entweder der ArbeitgeberInnen oder der ArbeitnehmerInnen ausgegangen.

\footnotetext{
${ }^{6}$ Die Lage der Arbeitszeit außerhalb der Hauptarbeitszeit (Abend, Nacht, Wochenende) wurde in insgesamt fünf Stufen erhoben. Diese reichen von „mehrmals pro/jede Woche“, „mehrmals im Monat“ über „einmal im Monat“ bis hin zu „seltener“ und „nie“.
} 
Als zentrale Dimension für die Zuordnung zu den einzelnen Formen werden Fragen des Einflusses von ArbeitnehmerInnen und ArbeitgeberInnen auf die Gestaltung der Arbeitszeit hinsichtlich Lage und Dauer betrachtet. Hinzu kommt der Aspekt der Planbarkeit der Arbeitszeiten, werden Arbeitszeiten von ArbeitgeberInnen kurzfristig geändert, wird die Flexibilität jedenfalls von dem/der ArbeitgeberIn bestimmt. Auf der anderen Seite kann, wenn für die Gestaltung der Arbeitszeit von ArbeitnehmerInnen in Gleitzeit häufig persönliche Bedürfnisse bestimmend sind, von arbeitnehmerInnenseitiger Gestaltung gesprochen werden.

Um auch indifferente, wenig flexible Situation beschreiben zu können, wird zusätzlich eine weitere Kategorie für insgesamt geringe Flexibilität eingeführt. Dies trifft vor allem im Bereich der Schichtarbeit mit langfristig gleichbleibenden Schichten zu, aber auch bei regelmäßig gleichen Diensten ohne Schwankungen.

Flexibilität im Sinne der ArbeitgeberInnen bedeutet hingegen häufig längere Arbeitszeiten, kurzfristige Verfügbarkeit oder kurzfristig wechselnde Dienstpläne. Flexibilität im Sinne der ArbeitnehmerInnen wird als die Möglichkeit zur (Mit-)Gestaltung der Arbeitszeiten und zur Planungssicherheit im Sinne der Einhaltung der mindestens zweiwöchigen Vorankündigungsfrist verstanden. Um den verschiedenen, empirisch erfassten Arbeitszeitmodellen gerecht zu werden, ist es also nötig, die Frage der Gestaltung der Arbeitszeiten modell-spezifisch zu operationalisieren.

In Übersicht 1 wird die konkrete Vorgangsweise bei der Operationalisierung dieser drei Formen von Arbeitszeitflexibilisierung für die einzelnen Arbeitszeitmodelle beschrieben. Die Zuordnung speist sich aus einer Reihe von Fragen, die die Mitbestimmung bei der Lage der Arbeitszeit, den Ankündigungsfristen der Arbeitszeiten und insbesondere das Auftreten kurzfristiger Änderungen betreffen. 


\section{Übersicht 1: Überblick über Zuordnung zu den Formen flexibler Arbeitszeitgestaltung}

\begin{tabular}{|c|c|c|c|}
\hline & $\begin{array}{l}\text { Beschäftigte mit stärker } \\
\text { arbeitnehmerInnenseitig } \\
\text { bestimmter Arbeitszeitfle- } \\
\text { xibilität }\end{array}$ & $\begin{array}{l}\text { Beschäftigte mit geringer } \\
\text { Arbeitszeitflexibilität }\end{array}$ & $\begin{array}{l}\text { Beschäftigte mit stärker arbeit- } \\
\text { geberInnenseitig bestimmter } \\
\text { Arbeitszeitflexibilität }\end{array}$ \\
\hline $\begin{array}{l}\text { Jede Woche } \\
\text { gleiche Ar- } \\
\text { beitsstunden }\end{array}$ & $\begin{array}{l}\text { Beschäftigte mit gleich blei- } \\
\text { benden Arbeitszeiten, die die } \\
\text { Lage ihrer Arbeitszeit weit- } \\
\text { gehend selbst bestimmen } \\
\text { können. }\end{array}$ & $\begin{array}{l}\text { Beschäftigte mit gleich blei- } \\
\text { benden Arbeitszeiten, deren } \\
\text { Arbeitszeit sowohl das } \\
\text { Ausmaß als auch die Lage } \\
\text { betreffend fixiert ist. }\end{array}$ & $\begin{array}{l}\text { Beschäftigte deren Verteilung der } \\
\text { Arbeitszeit auf die Arbeitswoche } \\
\text { von den ArbeitgeberInnen festge- } \\
\text { legt wird. Auch Beschäftigte mit } \\
\text { wiederholten kurzfristigen } \\
\text { Dienstplanänderungen durch die } \\
\text { ArbeitgeberInnen. }\end{array}$ \\
\hline $\begin{array}{l}\text { Schwankende } \\
\text { Arbeitszeit }\end{array}$ & $\begin{array}{l}\text { Beschäftigte, die die Vertei- } \\
\text { lung ihrer Arbeitszeit weit- } \\
\text { gehend selbst bestimmen } \\
\text { können. }\end{array}$ & $\begin{array}{l}\text { Aufgrund der jedenfalls } \\
\text { wechselnden Natur der } \\
\text { Arbeitszeiten erfolgt keine } \\
\text { Zuordnung. }\end{array}$ & $\begin{array}{l}\text { Beschäftigte, deren Arbeitszeit } \\
\text { durch Dienstpläne oder Verein- } \\
\text { barung mit den Arbeitgeber- } \\
\text { Innen festgelegt wird. Auch Be- } \\
\text { schäftigte, deren Arbeitszeiten } \\
\text { von ArbeitgeberInnen immer } \\
\text { wieder kurzfristig verändert wer- } \\
\text { den, finden sich in dieser Grup- } \\
\text { pe. }\end{array}$ \\
\hline $\begin{array}{l}\text { Regelmäßige } \\
\text { Schichten }^{\star} \text { ) }\end{array}$ & $\begin{array}{l}\text { Da Schichtpläne für Arbeit- } \\
\text { nehmerInnen jedenfalls mit } \\
\text { geringer Flexibilität verbun- } \\
\text { den sind, erfolgt hier keine } \\
\text { Zuordnung. }\end{array}$ & $\begin{array}{l}\text { Beschäftigte in regel- } \\
\text { mäßigen Schichten, die } \\
\text { mindestens zwei Wochen } \\
\text { im Voraus wissen, zu wel- } \\
\text { cher Schicht sie eingeteilt } \\
\text { sind. }\end{array}$ & $\begin{array}{l}\text { Beschäftigte, die weniger als zwei } \\
\text { Wochen im Voraus wissen, zu } \\
\text { welcher Schicht sie eingeteilt } \\
\text { sind. }\end{array}$ \\
\hline Gleitzeit $^{7}$ & $\begin{array}{l}\text { Beschäftigte, die mindestens } \\
\text { zwei von drei Motiven der } \\
\text { Gestaltung der Arbeitszeit } \\
\text { (eigene Arbeitszeit- } \\
\text { präferenzen, private Ver- } \\
\text { pflichtungen, private Inter- } \\
\text { essen) öfter als selten berück- } \\
\text { sichtigen. }\end{array}$ & $\begin{array}{l}\text { Gleitzeitvereinbarungen } \\
\text { lassen besonders viel Gestal- } \\
\text { tungsspielraum offen, aus } \\
\text { diesem Grund erfolgt hier } \\
\text { keine Zuordnung. }\end{array}$ & $\begin{array}{l}\text { Befragte, die mindestens zwei } \\
\text { von drei Motiven der Gestaltung } \\
\text { der Arbeitszeit (eigene Arbeits- } \\
\text { zeitpräferenzen, private Ver- } \\
\text { pflichtungen, private Interessen) } \\
\text { bei der Gestaltung ihrer Arbeits- } \\
\text { zeiten selten oder gar nicht be- } \\
\text { rücksichtigen. }\end{array}$ \\
\hline
\end{tabular}

*) Obwohl Beschäftigte in regelmäßigen Schichten in manchen Fällen in die Dienstplangestaltung eingebunden sind, bzw. dort die Möglichkeit zur Mitsprache haben, wird hier davon ausgegangen, dass Schichtarbeit den ArbeitnehmerInnen grundsätzlich wenig (kurzfristige) Flexibilität einräumt, abgesehen vielleicht von individuell vereinbartem Schichttausch mit KollegInnen.

\footnotetext{
${ }^{7}$ Beschäftigte in Gleitzeitmodellen wurden nach den Motivlagen für die Gestaltung ihrer Arbeitszeit gefragt. Diese erfassten Themen reichen von Anordnungen der Vorgesetzten, über die Arbeitsmenge bis zu persönlichen Motiven wie Betreuungsverpflichtungen, eigenen Bedürfnissen oder privaten Interessen.
} 


\section{Flexibilität in den Branchen}

In einem ersten Schritt wird nun diskutiert, wie sich die einzelnen untersuchten Branchen in Hinblick auf diese drei Formen flexibler Gestaltung der Arbeitszeiten unterscheiden.

Von arbeitnehmerInnenseitiger Flexibilität wird innerhalb der betrachteten Branchen am häufigsten aus der IT berichtet. Hier wird die Arbeitszeit von 49\% der Beschäftigten überwiegend von ihren Interessen gesteuert. $\mathrm{Zu}$ jeweils etwa einem Viertel ordnen sich Beschäftigte aus der Elektroindustrie (27\%) und aus dem Bereich der Banken (24\%) hier zu. Am geringsten ist dieser Anteil mit 16\% in der Gastronomie. In der Gastronomie, geben 42\% der Beschäftigten an, dass ihre Arbeitszeiten vorwiegend von den Flexibilitätsanforderungen der ArbeitgeberInnenseite bestimmt sind. In den anderen Branchen liegen diese Werte zwischen $26 \%$ in der Elektroindustrie, 32\% im Handel und 36\% in der IT. Insgesamt geringe Flexibilität der Arbeitszeiten finden sich besonders häufig bei ArbeitnehmerInnen in der Reinigung mit 55\% und im Metallsektor mit 54\% vor. Mit Ausnahme der IT sind auch in den anderen untersuchten Branchen etwas weniger als die Hälfte der ArbeitnehmerInnen mit wenig flexiblen Arbeitszeiten konfrontiert. Einzig in der IT liegt dieser Anteil bei nur 15\%.

Abbildung 1: Formen flexibler Arbeitszeitgestaltung in den Branchen.

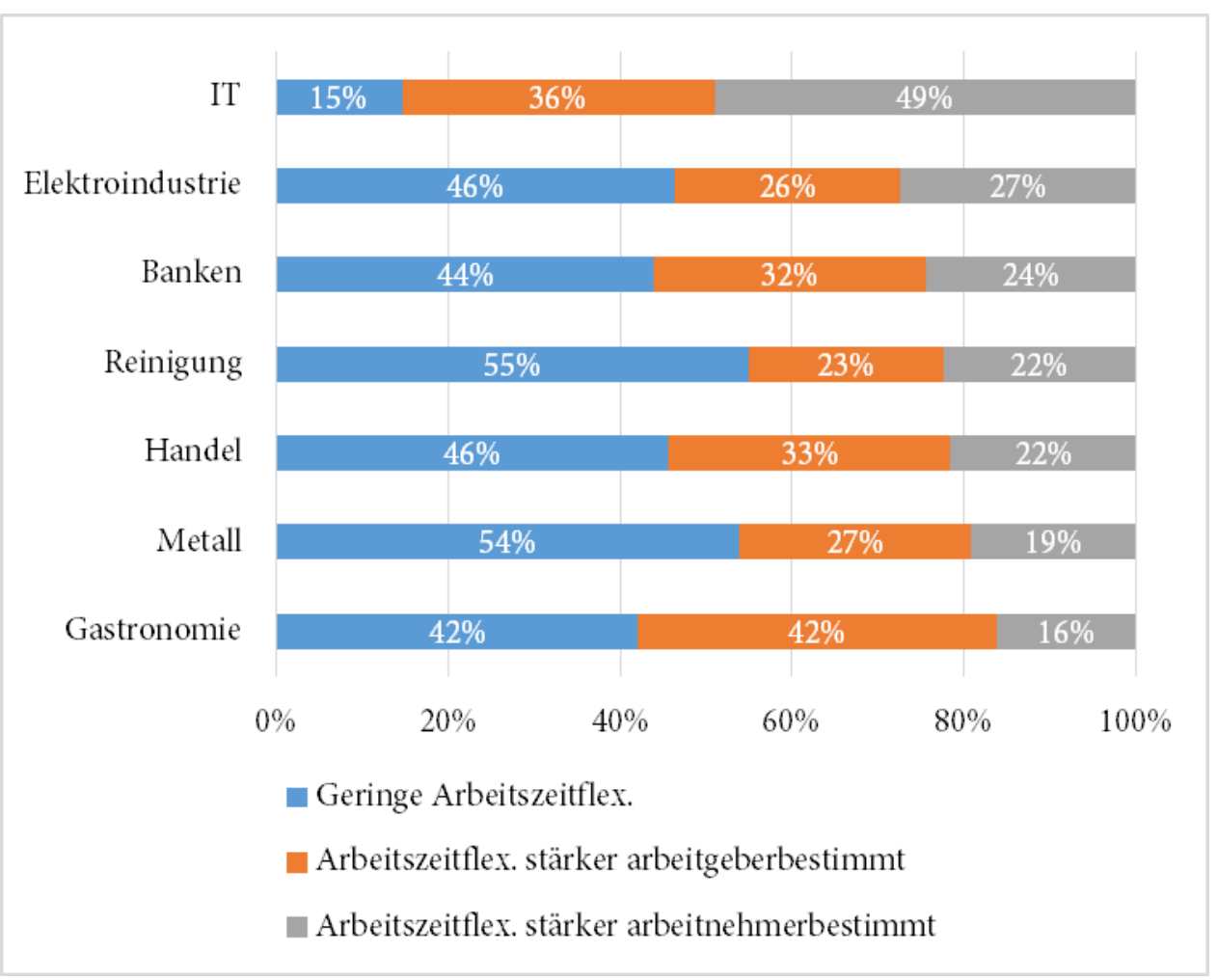

Quelle: FORBA-Erhebung zu Arbeitszeitflexibilisierung 2015.

Quer über die Branchen zeigt sich ein deutlicher Zusammenhang zwischen Arbeitszeitgestaltung und Bildungsabschluss. Je höher der erreichte formale Bildungsgrad, desto höher ist der Anteil von ArbeitnehmerInnen, die ihre Arbeitszeit in ihrem eigenen Sinne, d.h. arbeitnehmer Innenbestimmt (mit-)gestalten können. Bei den AbsolventInnen einer Universität oder einer Fachhochschule können etwas mehr als die Hälfte der ArbeitnehmerInnen (51\%) ihre Ar- 
beitszeit vorwiegend selbst bestimmen. Je niedriger der formale Bildungsgrad der befragten Personen, umso höher liegt der Anteil an ArbeitnehmerInnen, die über insgesamt geringe Flexibilität verfügen. Bei den AbsolventInnen einer Pflichtschule und einer Lehre sind dies mit 57\% bzw. 50\% jeweils mindestens die Hälfte. Auch der Anteil der ArbeitnehmerInnen, deren Arbeitszeitflexibilität vorwiegend von ArbeitgeberInnen bestimmt ist, ist bei niedrigerer Bildung deutlich höher. Bei PflichtschulabsolventInnen findet sich etwas mehr als ein Viertel der ArbeitnehmerInnen in dieser Gruppe, ebenfalls noch recht hoch ist dieser Anteil bei den AbsolventInnen einer Lehre mit 30\%.

Abbildung 2: Formen flexibler Arbeitszeitgestaltung nach höchster abgeschlossener Ausbildung.

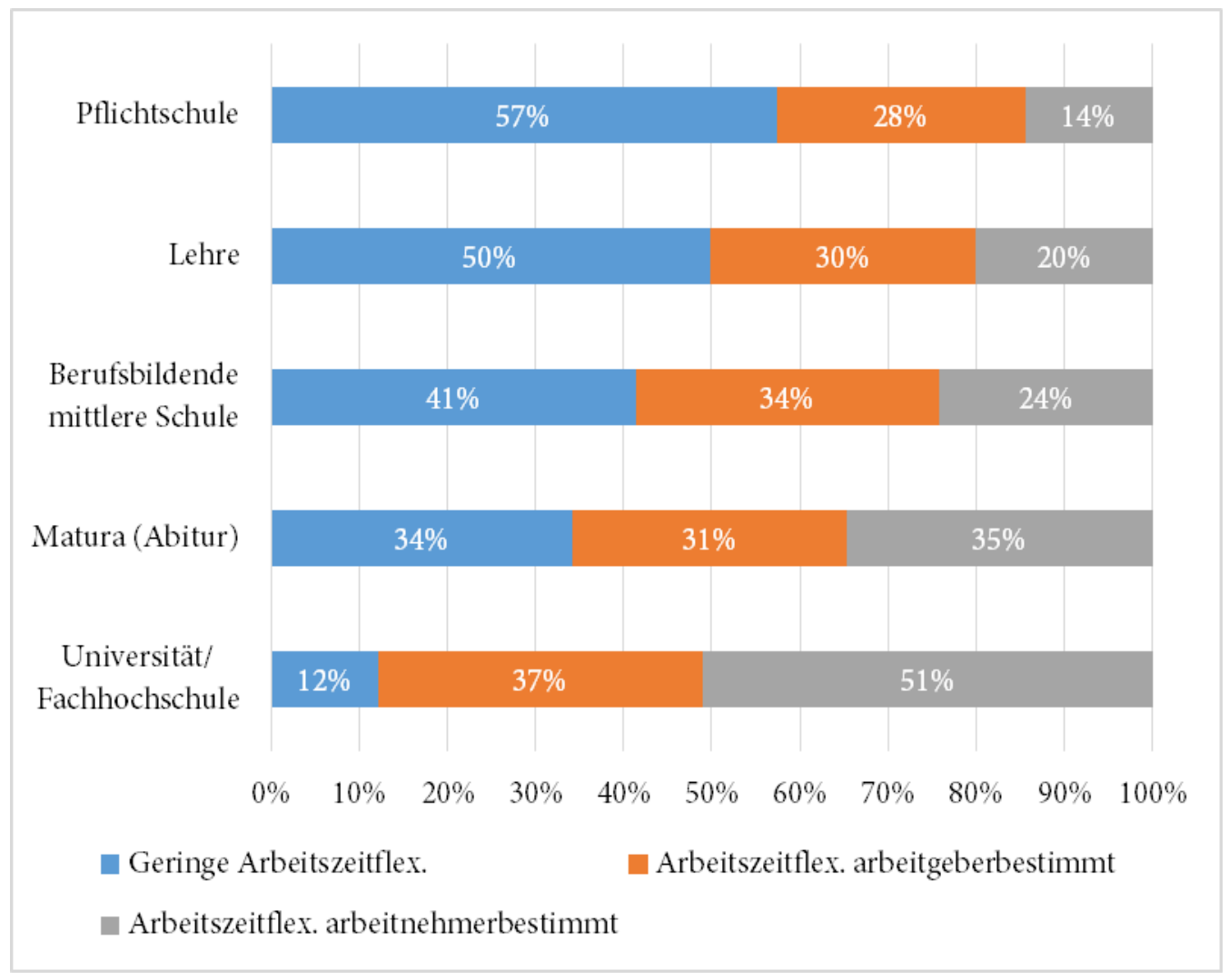

Quelle: FORBA-Erhebung zu Arbeitszeitflexibilisierung 2015.

Eng verbunden mit der Frage flexibler Arbeitszeiten und deren Gestaltung ist die Frage nach Über- und Mehrstunden und der Abgeltung dieser zusätzlich gearbeiteten Stunden. Gerade flexible Arbeitszeiten eröffnen Möglichkeiten zur Ausdehnung von Arbeitszeiten, insbesondere wenn diese von Interessen von ArbeitgeberInnen getrieben werden. So können durch zusätzlich gearbeitete Stunden der MitarbeiterInnen, neben der allgemeinen Erhöhung des Arbeitsoutputs durch den Einsatz von mehr Stunden, auch kurzfristige Schwankungen des Arbeitsanfalls ausgeglichen oder Defizite in der Planung des Arbeitseinsatzes kompensiert werden. 


\section{Über- und Mehrstunden}

Über- und Mehrstunden sind für viele ArbeitnehmerInnen Teil des Arbeitsalltags, das zeigen die Ergebnisse der Befragung deutlich. Jede Woche erbringt etwas mehr als ein Viertel (28\%) der befragten ArbeitnehmerInnen Über- oder Mehrstunden, mehrmals monatlich tun dies $21 \%$. Noch deutlich darüber liegen Erwerbstätige mit Gleitzeitmodell. Hier geben $43 \%$ der Beschäftigten an wöchentlich Mehrleistungen zu erbringen und 26\% berichten mindestens monatlich mehr Stunden als die vertragliche Arbeitszeit zu arbeiten.

Im Gegensatz zu diesen Gruppen mit häufigen Mehrstunden kommen zusätzlich gearbeitete Stunden bei Beschäftigte mit jede Woche gleichen Stunden zu 23\% nie und zu weiteren $24 \%$ weniger als einmal monatlich vor. Beschäftigte, deren Arbeitszeit in Schichten organisiert ist, leisten zu 19\% nie und zu 24\% weniger als einmal monatlich Mehrstunden.

\section{Abbildung 3: Häufigkeit von Mehrstunden nach Arbeitszeitmodell.}

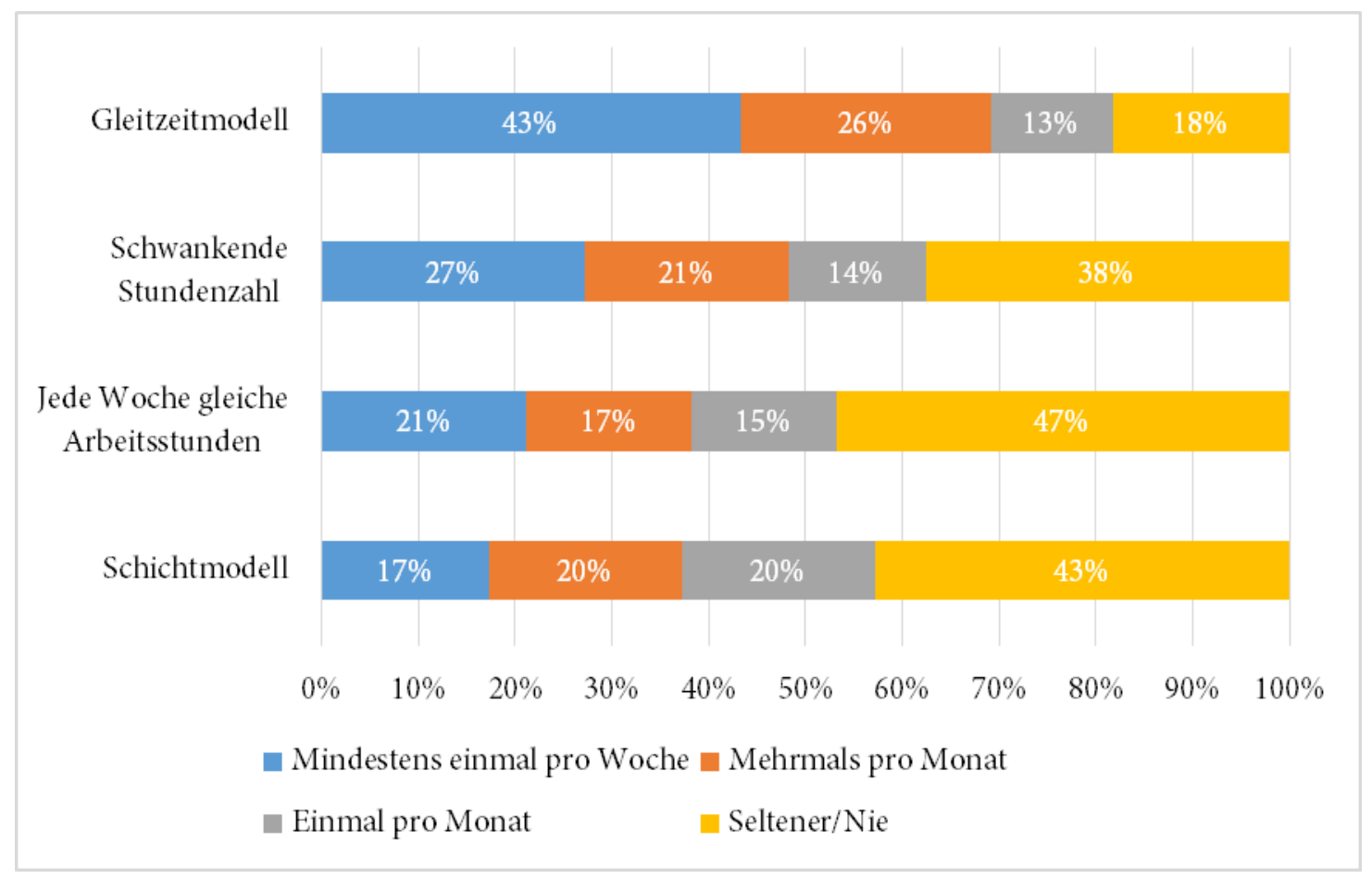

Quelle: FORBA-Erhebung zu Arbeitszeitflexibilisierung 2015.

Auch hier zeigt sich wieder ein deutlicher Zusammenhang mit dem Bildungsgrad. AbsolventInnen einer Universität oder einer Allgemeinbildenden oder Berufsbildenden Höheren Schule (AHS/BHS) leisten regelmäßig Überstunden. Im Gegensatz dazu leisten 51\% der PflichtschulabsolventInnen selten oder nie Überstunden. Bei den LehrabsolventInnen sind dies immerhin noch 40\% und bei den AbsolventInnen einer BMS 37\%. 
Abbildung 4: Häufigkeit von Mehrstunden nach höchster abgeschlossener Ausbildung.

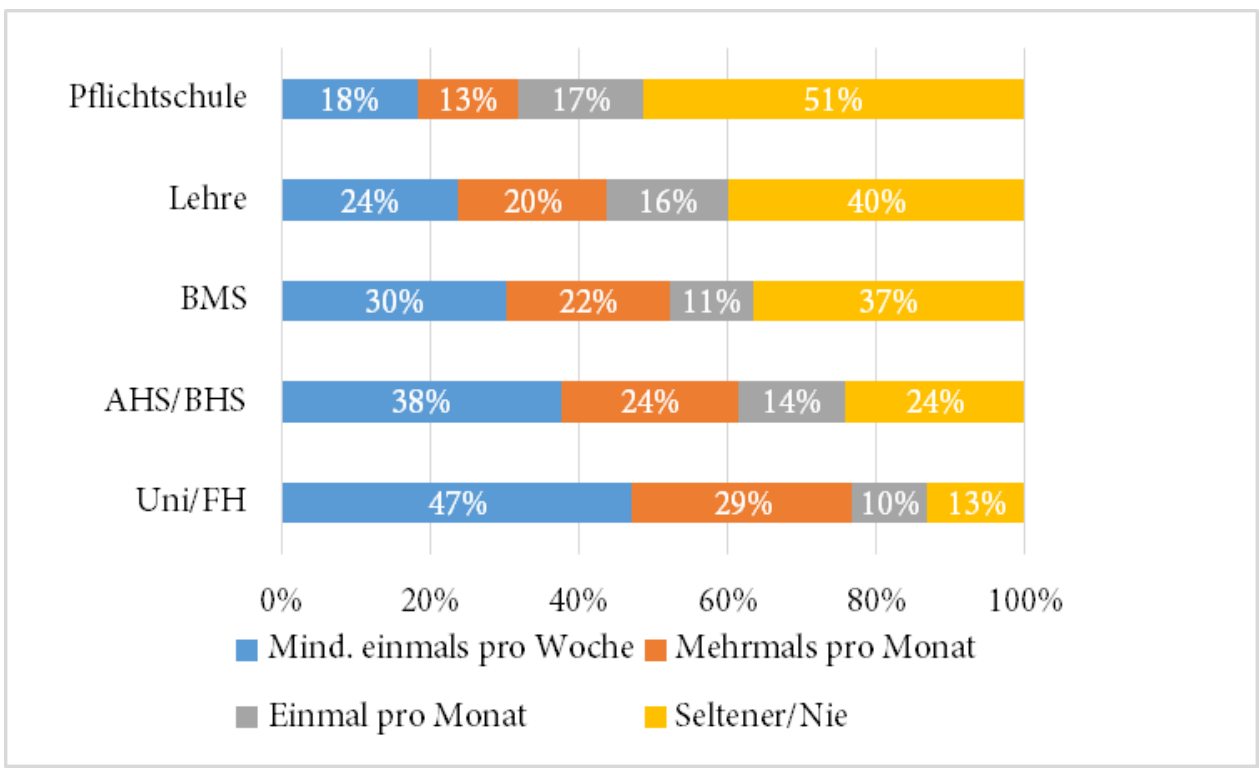

Quelle: FORBA-Erhebung zu Arbeitszeitflexibilisierung 2015.

\section{Abgeltung von Über- und Mehrstunden}

Mit der Frage der Leistung von Mehrstunden eng verknüpft ist jene nach deren Abgeltung. Über die vereinbarte Wochenarbeitszeit hinaus geleistete Arbeitsstunden werden häufig in irgendeiner Form ausgeglichen. Bei Beschäftigten mit einer Gleitzeitvereinbarung geschieht dies vorwiegend in Form von Zeitausgleich, der je nach betrieblicher Vereinbarung in einzelnen Stunden oder auch in ganzen freien Tagen konsumiert werden kann. Zuschläge sind für Mehrstunden, wenn sie eine bestimmte Grenze nicht überschreiten, nicht vorgesehen. Jedoch erleben ArbeitnehmerInnen mit Gleitzeitvereinbarung immer wieder, dass angesammelte Plusstunden am Ende einer Durchrechnungsperiode gestrichen werden. Gelingt der Abbau von zusätzlichen Arbeitsstunden im Gleitzeitmodell nicht durch Zeitausgleich, ist auch eine Abgeltung durch Überstunden möglich.

ArbeitnehmerInnen mit Mehrstunden werden immer wieder die auf Über- und Mehrstunden entfallenden Zuschläge nicht bezahlt bzw. Zeitausgleich nur ohne Zuschlag gewährt. Dies ist jedoch nicht in allen Arbeitszeitmodellen tatsächlich zulässig. So werden Überstunden nicht oder nicht vollständig abgegolten. Abbildung 6 gibt einen Überblick über die Praxis der Überstundenabgeltung in den sieben untersuchten Branchen.

Knapp zwei Dritteln (62\%) der befragten ArbeitnehmerInnen werden ihre Mehrleistungen abgegolten. 23\% erhalten Zeitausgleich oder Bezahlung der Über- und Mehrstunden ohne Zeitausgleich. Weitere 5\% erhalten diese Abgeltung nur zum Teil mit Zuschlag. Insgesamt 7\% der Befragten berichten von insgesamt unbezahlter Mehrarbeit oder gestrichenen Plusstunden. 3\% der ArbeitnehmerInnen werden im Rahmen eines All-In-Vertrages oder einer Überstundenpauschale über diese Vereinbarung hinausgehende Überstunden nicht abgegolten. Insgesamt erhält somit etwas mehr als ein Drittel (38\%) der ArbeitnehmerInnen in den sieben untersuchten Branchen mit Über- oder Mehrstunden diese Leistungen nicht vollständig entgolten. 
Abbildung 5: Abgeltung von Mehrstunden.

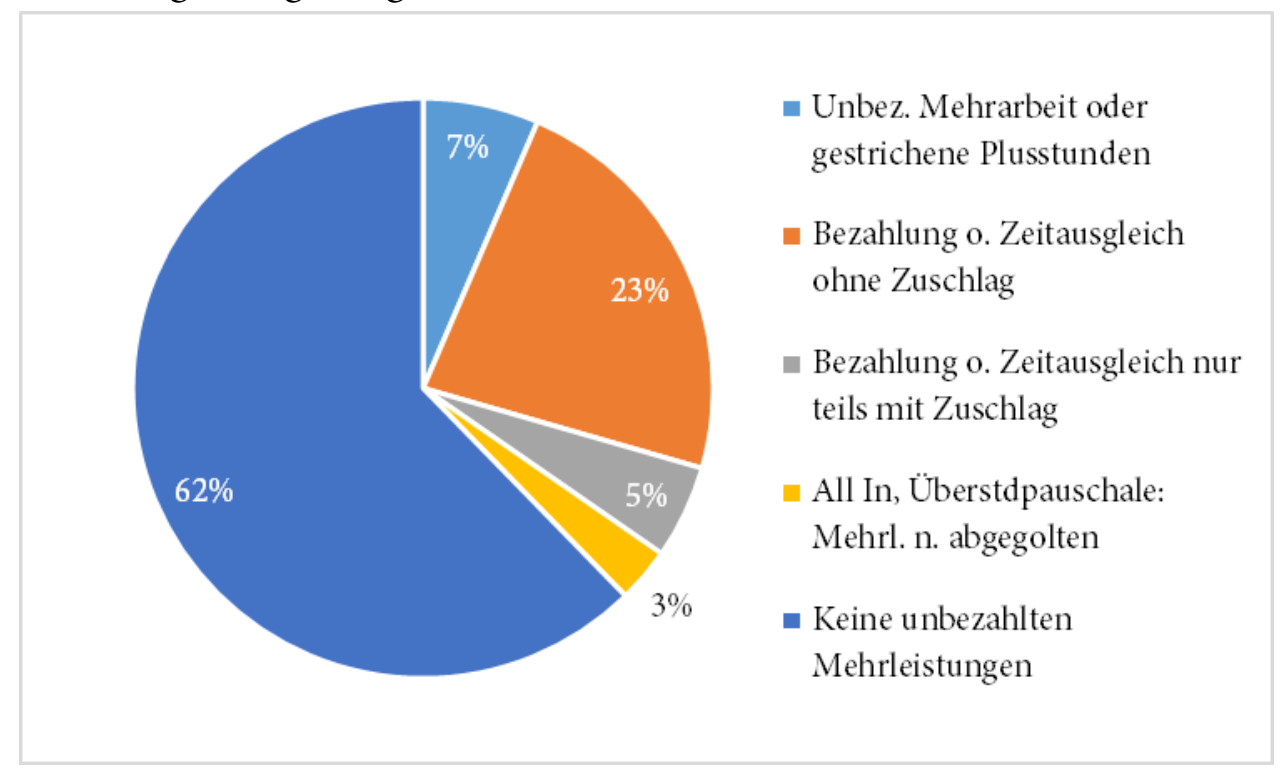

Quelle: FORBA-Erhebung zu Arbeitszeitflexibilisierung 2015.

\section{Faktoren für Mehrarbeit und die Nicht-Abgeltung dieser Arbeitsstunden}

Für eine vertiefende Analyse des Risikos für Mehrarbeit und unbezahlte Mehrarbeit werden im folgenden Abschnitt nun mit Hilfe zweier binär logistischer Regressionsmodelle verschiedene mögliche erklärende Faktoren zusammengeführt (Backhaus et al. 2016; Diaz-Bone 2017)

In einem ersten Modell werden Faktoren für ein erhöhtes Risiko für Über- und Mehrstunden ermittelt und in einem zweiten Modell für die Gruppe der Personen mit Über- oder Mehrstunden analysiert, welche Merkmale von ArbeitnehmerInnen das Risiko für (die Chance auf) nicht abgegoltene Mehrarbeit erhöhen. In beide Modelle wurden die gleichen unabhängigen Variablen aufgenommen. Neben dem Geschlecht und der Frage, ob in Vollzeit oder in Teilzeit gearbeitet wird, wurden die folgenden Einflussfaktoren getestet: Nachdem die Befragung insgesamt sieben Branchen umfasst hat, muss untersucht werden, welchen Einfluss die jeweilige Branche auf das Risiko für Mehrarbeit und darauffolgend für unbezahlte Mehrarbeit aufweisen. Ein wichtiger möglicher Einflussfaktor ist das Arbeitszeitmodell, d.h. die eingangs deskriptiv beschriebene Frage, ob jede Woche die gleichen Stunden gearbeitet werden, ob die Anzahl der Wochenstunden schwankt, ob ein Gleitzeitmodell vereinbart wurde oder ob in regelmäßigen Schichten gearbeitet wird.

Ebenfalls in beiden logistischen Regressionsmodellen enthalten ist die höchste abgeschlossene Ausbildung der Befragten. Die deskriptive Analyse hat gezeigt, dass es je nach Bildungsabschluss große Unterschiede bei der Leistung von Mehrstunden gibt. Mehrarbeit tritt häufiger bei höher qualifizierten ArbeitnehmerInnen auf. Schließlich wird untersucht, ob ein Einfluss der Form der Gestaltung flexibler Arbeitszeiten feststellbar ist. Hier wird wieder die weiter oben entwickelte Unterscheidung zwischen arbeitsgeberInnenseitiger, arbeitnehmerInnenseitiger oder insgesamt geringer Flexibilität der Arbeitszeiten herangezogen. Da bei der For- 
mulierung der Arbeitszeit-Modelle und bei der Zuordnung dieser Modelle zu den verschiedenen Flexibilitätsgruppen nicht nach der Lage der Arbeitszeit differenziert wurde, wird diese Information ebenfalls in die Überprüfung möglicher Einflussfaktoren aufgenommen. Tabelle 5 gibt einen Überblick über die Ergebnisse.

Tabelle 3: Einflussfaktoren auf das Risiko für Mehrarbeit und unbezahlte Mehrarbeit

\begin{tabular}{|c|c|c|}
\hline & $\begin{array}{l}\text { Risiko für Mehrar- } \\
\text { beit }\end{array}$ & $\begin{array}{l}\text { Risiko für unbezahl- } \\
\text { te Mehrarbeit (nur } \\
\text { Personen mit Mehr- } \\
\text { oder Plusstunden) }\end{array}$ \\
\hline & $\operatorname{Exp}(B)$ & $\operatorname{Exp}(B)$ \\
\hline Geschlecht (Frauen) & $0.666^{* * *}$ & 0.944 \\
\hline Teilzeit & $0.369^{* * *}$ & $1.547^{* * *}$ \\
\hline \multicolumn{3}{|l|}{ Branche, Referenz: Handel } \\
\hline Metall (Industrie oder Gewerbe) & 1.037 & $0.618^{\star}$ \\
\hline Elektroindustrie & 0.819 & $0.615^{\star}$ \\
\hline Gastronomie und Beherbergung & 1.186 & $1.655^{*}$ \\
\hline IT & 0.823 & 0.803 \\
\hline Banken & 0.779 & $1.668^{\star *}$ \\
\hline Reinigung & $0.590^{*}$ & 1.166 \\
\hline \multicolumn{3}{|l|}{ Arbeitszeitmodell, Referenz: Jede Woche gleiche Arbeitsstunden } \\
\hline Schwankende Stundenanzahl & $1.554^{\star}$ & 1.247 \\
\hline Gleitzeit & 1.288 & $0.246^{* * *}$ \\
\hline Regelm. Schichten & $1.465^{\star *}$ & $0.602^{* *}$ \\
\hline \multicolumn{3}{|l|}{ Bildung, Referenz: Uni/FH } \\
\hline Pflichtschule & $0.359^{* * *}$ & 0.796 \\
\hline Lehre & $0.384^{* * *}$ & $0.482^{* * *}$ \\
\hline BMS & $0.551^{\star *}$ & $0.442^{\star * \star}$ \\
\hline AHS/BHS & $0.602^{\star *}$ & $0.502^{* * *}$ \\
\hline \multicolumn{3}{|l|}{ Form der Arbeitszeitflexibilisierung, Referenz: geringe AZ-Flex } \\
\hline $\begin{array}{l}\text { Arbeitszeitflexibilität überwiegend arbeitgeberInnenbe- } \\
\text { stimmt }\end{array}$ & $1.838^{\star \star}$ & 0.778 \\
\hline $\begin{array}{l}\text { Arbeitszeitflexibilität überwiegend arbeitnehmerInnenbe- } \\
\text { stimmt }\end{array}$ & $1.700^{* *}$ & 0.981 \\
\hline Arbeit am Samstag & $2.083^{* * *}$ & 0.907 \\
\hline Arbeit am Sonntag & 0.850 & 0.909 \\
\hline Arbeit am Abend & $1.588^{* * *}$ & 1.024 \\
\hline Arbeit in der Nacht & 0.815 & 0.761 \\
\hline Konstante & 1.162 & 2.677 \\
\hline
\end{tabular}

Quelle: FORBA-Erhebung zu Arbeitszeitflexibilisierung 2015. ${ }^{* *} p<0.01,{ }^{* *} p<0.05,{ }^{\star} p<0.10$

\section{Einflussfaktoren auf das Risiko für Mehrarbeit}

Frauen haben, verglichen mit Männern, ein etwa ein Drittel geringeres Risiko für Mehrarbeit. Arbeiten Frauen oder Männer in Teilzeit, reduziert sich dieses Risiko um etwas mehr als die 
Hälfte. Auf die Branchen entfällt mit Ausnahme der Reinigung kein signifikanter Einfluss. In der Reinigung ist das Risiko für Mehrarbeit etwas mehr als halb so groß.

Von den vier unterschiedenen Arbeitszeitmodellen weisen Beschäftigte mit schwankenden Stunden und in regelmäßigen Schichten jeweils ein um die Hälfte höheres Risiko für Mehrarbeit als Beschäftigte in der Referenzkategorie "Jede Woche gleiche Stunden“ auf. Auch die höchste abgeschlossene Ausbildung beeinflusst, wie schon in der deskriptiven Analyse sichtbar wurde, die Chance auf Mehrarbeit deutlich. Verglichen mit AbsolventInnen einer Universität oder einer FH, weisen AbsolventInnen von Pflichtschule, einer Lehre und einer Berufsbildenden mittleren Schule (BMS) ein signifikant niedrigeres Risiko für Mehrstunden auf.

Im Vergleich zu insgesamt geringer Arbeitszeitflexibilisierung erhöhen arbeitgeberinnenseitige und arbeitnehmerInnenseitige Flexibilisierung das Risiko für Mehrarbeit deutlich, der Effekt arbeitgeberseitiger Flexibilisierung ist noch etwas stärker als der Effekt arbeitnehmerInnenseitiger Flexibilisierung. Vor allem Arbeit am Abend und Arbeit am Samstag erhöht das Risiko auf Mehrarbeit. Dieser Effekt ist naheliegend, da lange Arbeitszeiten nahezu zwangsläufig zu Arbeit an den Rändern des Tages oder am Wochenende führen müssen.

\section{Einflussfaktoren auf das Risiko für unbezahlte Mehrarbeit}

In einem nächsten Schritt wurde für die Gruppe der Personen mit Mehrarbeitsstunden untersucht, welche Merkmale das Risiko erhöhen, dass diese Arbeitsstunden gar nicht oder nur zum Teil abgegolten werden. Die Ergebnisse sind ebenfalls in Tabelle 3 dargestellt. Hier werden sowohl Personen erfasst, deren Mehrarbeitsstunden vollständig nicht bezahlt werden als auch Personen, deren zusätzlich gearbeitete Stunden zum Teil nicht entgolten werden oder deren Plusstunden im Gleitzeitmodell zum Teil oder vollständig gestrichen werden.

Im Gegensatz zur Frage nach der Mehrarbeit geht bei der Frage der Abgeltung von Mehrarbeitsstunden vom Geschlecht kein signifikanter Einfluss aus. Allerdings haben Teilzeitbeschäftigte, und damit doch wiederum auch viele Frauen, ein um die Hälfte erhöhtes Risiko, dass Mehrstunden nicht oder nicht vollständig bezahlt werden.

Nach Branchen betrachtet, gibt es in der Gastronomie, verglichen mit dem Handel als Referenz, ein um zwei Drittel erhöhtes Risiko und im Metallsektor und der Elektroindustrie ein signifikant niedrigeres Risiko auf unbezahlte Mehrarbeit.

Gleitzeitbeschäftigte und Beschäftigte in regelmäßigen Schichten weisen, verglichen mit Beschäftigten mit jede Woche gleichbleibenden Stunden, ein deutlich niedrigeres Risiko für nicht-entgoltene Mehrarbeit auf. Beim Arbeitszeitmodell Gleitzeit reduziert sich dieses Risiko auf ein Viertel. Besonders viele Beschäftigte in Gleitzeitmodellen leisten immer wieder Mehrstunden, das hat die Analyse zu den Mehrstunden ergeben. Zugleich können diese Stunden in vielen Fällen wohl durch weniger gearbeitete Stunden wieder ausgeglichen werden. Aus diesem Grund ist gerade für die große Gruppe der Gleitzeitbeschäftigten das Risiko für unbezahlte Mehrarbeit gering.

So wie bei der Frage nach Mehrarbeit, übt der höchste Bildungsabschluss auch großen Einfluss auf das Risiko für unbezahlte Überstunden auf. Besonders betroffen sind Beschäftigte mit sehr hoher Qualifikation. Verglichen mit Uni- bzw. FH-AbsolventInnen haben (mit Ausnahme der Pflichtschule) alle Bildungsgruppe ein signifikant niedrigeres Risiko auf unbezahlte Mehrstunden. 
Die Form der Flexibilisierung der Arbeitszeiten, ob durch ArbeitgeberInnen oder durch ArbeitnehmerInnen bestimmt oder insgesamt gering ausgeprägt, übt keinen signifikanten Einfluss auf das Risiko für nicht bezahlte Arbeitsstunden aus. Ebenfalls keinen signifikanten Effekt auf das Risiko für unbezahlte Arbeit hat die Lage der Arbeitszeit. Hier dominieren Effekte der Branchen. Wenn beispielsweise im Rahmen von Schichtplänen die Arbeitsleistung am Abend, in der Nacht oder am Wochenende erbracht wird, wird diese meist auch entsprechend entlohnt.

Die Merkmalskonstellationen, die zu Mehrarbeit oder zu unbezahlter Mehrarbeit führen, unterscheiden sich somit. Das Risiko für Mehrarbeit und damit unbezahlte Mehrarbeit ist eng verknüpft mit dem Bildungsgrad und damit wohl mit der nicht näher erhobenen beruflichen Tätigkeit. Das Arbeitszeitmodell Gleitzeit führt jedoch nicht häufiger zu unbezahlten Arbeitsstunden. Teilzeitarbeit senkt das Risiko für Mehrarbeit, wird allerdings Mehrarbeit geleistet, wird diese bei Teilzeit häufig nicht (vollständig) abgegolten. ArbeitgeberInnen- und arbeitnehmerInnenseitige Flexibilisierung erhöhen das Risiko auf Mehrarbeit nicht aber auf unzureichende Abgeltung dieser.

Spiegeln sich diese Befunde auch in den Aussagen zur Zufriedenheit wieder? Wie werden die einzelnen Formen der Arbeitszeitgestaltung bewertet? Sind Beschäftigte mit arbeitnehmerseitig gestalteter Arbeitszeit weniger zufrieden als Beschäftigte, die ihre Arbeitszeit stärker selbst gestalten können?

\section{Zufriedenheit mit dem Arbeitszeitmodell}

Gleitzeitbeschäftigte arbeiten, wie weiter oben gezeigt wurde, besonders häufig über die vereinbarten Wochenstunden hinaus, zugleich geben Beschäftigte mit diesem Modell die höchste Zufriedenheit an. ${ }^{8}$ So sind 76\% der Frauen mit Gleitzeit sehr und weitere 22\% ziemlich zufrieden mit ihrem Arbeitszeitmodell (vgl. Abbildung ). Männer liegen etwas darunter, von diesen sind 68\% der Befragten sehr und 27\% ziemlich zufrieden. Nachdem für Gleitzeitbeschäftigte das Risiko für unbezahlte Mehrarbeit nicht erhöht ist, dürfte in vielen Fällen ein Ausgleich dieser Mehrarbeitsstunden gelingen.

\footnotetext{
${ }^{8}$ Die Zufriedenheit mit dem Arbeitszeitmodell wurde auf einer vierstufigen Skala erhoben, diese reichte von „sehr zufrieden“, „ziemlich zufrieden“, „wenig zufrieden“ und „gar nicht zufrieden“.
} 
Abbildung 6: Zufriedenheit mit dem Arbeitszeitmodell nach Arbeitszeitmodell.

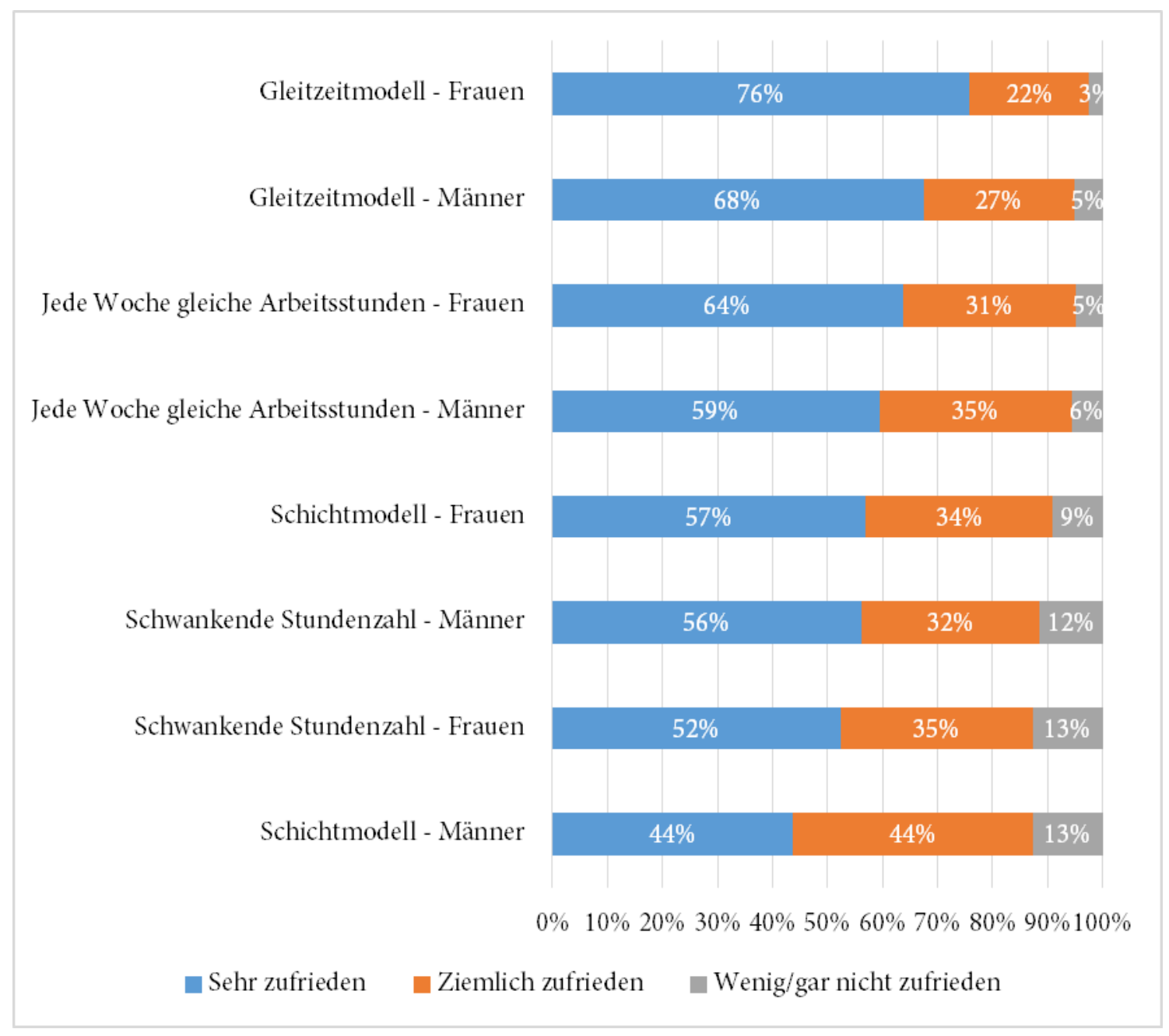

Quelle: FORBA-Erhebung zu Arbeitszeitflexibilisierung 2015.

An zweiter Stelle in der Reihung der Zufriedenheit mit dem Arbeitszeitmodell folgen jede Woche gleiche Stunden. Hier leisten vergleichsweise wenige Beschäftigte Mehrstunden, die Arbeitszeiten sind also wohl vorhersehbar und gut planbar, was zu guten Zufriedenheitswerten beitragen kann.

Regelmäßige Schichten bergen zwar geringes Risiko für unbezahlte Mehrarbeit, bieten aber geringe Flexibilität. Immer wieder sind die Schichten, da ja mehrere ArbeitnehmerInnen an einem Arbeitsplatz beschäftigt sind, an den Randzeiten des Tages oder in der Nacht angesiedelt. Dieser Umstand dürfte auch zu größerer Unzufriedenheit beitragen, besonders im Falle von Männern, die zu überwiegenden Teil ihre Vollzeitarbeit in einem Schichtmodell erbringen.

Schwankende Stundenanzahl findet sich besonders häufig in der Gastronomie und in der Reinigung. In dieser Branche ist das Risiko für Mehrarbeit oder unbezahlte Mehrarbeit nicht erhöht, gleichzeitig muss sich vor allem in dieser Branche die Arbeitszeit verstärkt an den Vorgaben des Arbeitsgebers ausrichten.

ArbeitgeberInnenseitige Flexibilisierung ist mit höherer Unzufriedenheit verbunden als insgesamt geringe Flexibilität der Arbeitszeiten. Nur 55\% der ArbeitnehmerInnen mit arbeit- 
geberInnenseitiger Flexibilität bezeichnen sich als sehr zufrieden und 34\% als ziemlich zufrieden. $11 \%$ ordnen sich den Antwortmöglichkeiten wenig oder gar nicht zufrieden zu. Etwas besser wird die Situation bei insgesamt geringer Flexibilität bewertet. Insgesamt am zufriedensten sind ArbeitnehmerInnen, die ihre Arbeitszeit zumindest zum Teil selbst gestalten können (arbeitnehmerInnenseitige Flexibilisierung). Hier sind 58\% sehr und 36\% ziemlich zufrieden.

Abbildung 7: Zufriedenheit mit dem Arbeitszeitmodell nach Form flexibler Arbeitszeiten.

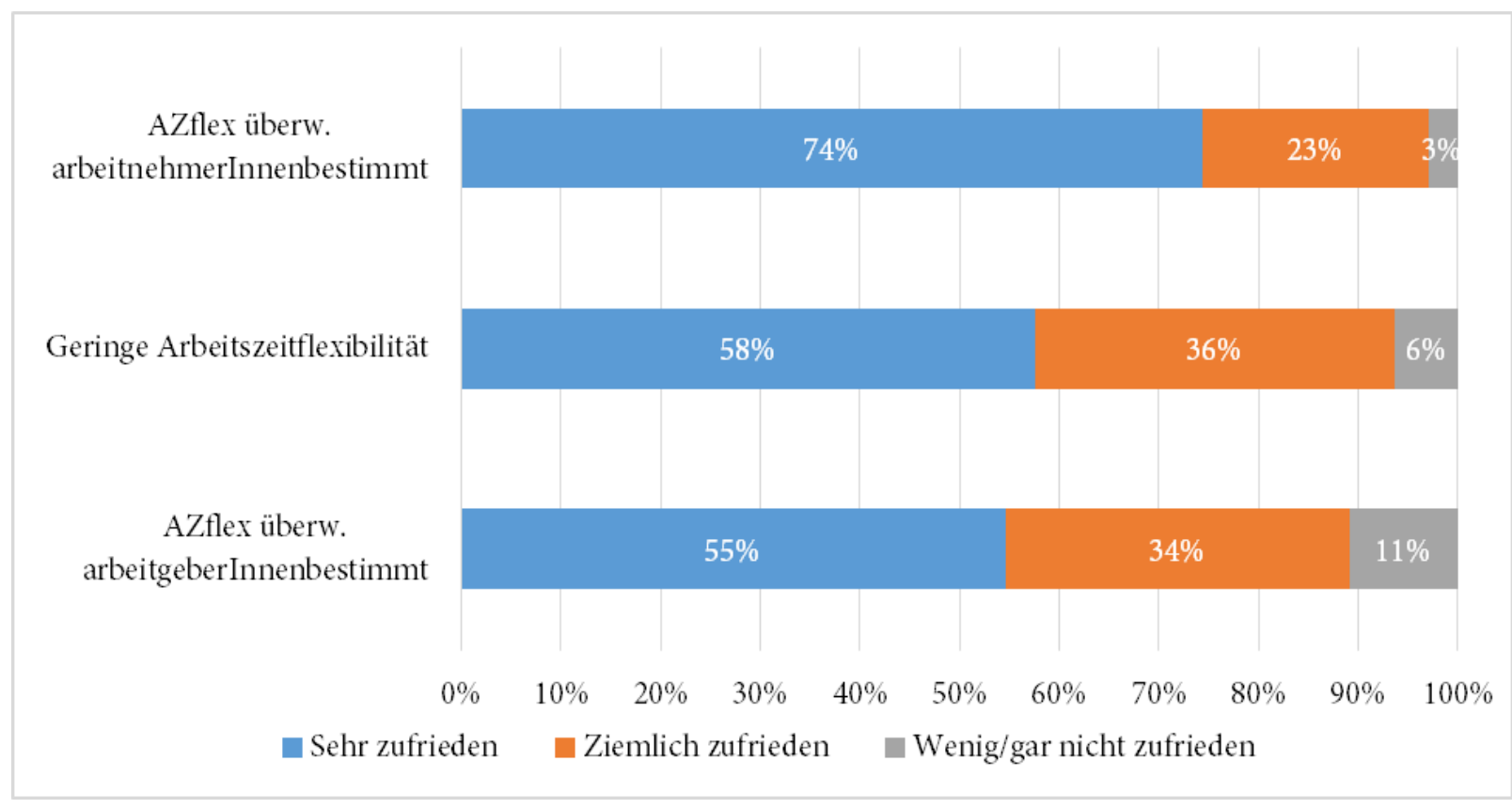

Quelle: FORBA-Erhebung zu Arbeitszeitflexibilisierung 2015.

\section{Schlussfolgerungen}

Mit den in diesem Beitrag präsentierten Analysen wurde auf Basis einer Befragung von ArbeitnehmerInnen in sieben ausgewählten österreichischen Branchen untersucht, wer flexible Arbeitszeitgestaltung vorwiegend antreibt, wie diese jeweils auf bezahlte und unbezahlte Mehrarbeitsstunden wirken und unter welchen Bedingungen flexible Arbeitszeiten von ArbeitnehmerInnen positiv und in welchen Fällen diese negativ bewertet werden. Die Untersuchung hat gezeigt, dass flexible Arbeitszeiten häufiger mit Mehrarbeit verbunden sind als insgesamt wenig flexible Arbeitszeiten. Zusätzlich leisten höher Gebildete häufiger Mehrarbeit. Wird am Abend oder am Samstag gearbeitet, ist dies ebenfalls häufig mit Mehrarbeit verbunden. Von diesen Zusammenhängen unterscheiden sich die Risikofaktoren für unbezahlte Mehrarbeit deutlich. Hier treten vor allem zwei Branchen, die Gastronomie und der Bankensektor, hervor. Zusätzlich haben geringer Gebildete ein signifikant höheres Risiko auf unbezahlte Mehrarbeit.

Trotz des vorhandenen Risikos für Mehrarbeit wird Flexibilisierung positiv bewertet, wenn die ArbeitnehmerInnen in die Gestaltung flexibler Arbeitszeiten eingebunden sind. Zusätzlich gearbeitete Stunden können (auch) im Interesse von ArbeitnehmerInnen sein, bei- 
spielsweise wenn diese zu für ArbeitnehmerInnen sinnvollen Zeiten wieder ausgeglichen werden können. Sind die Arbeitszeiten vorwiegend nach den Anforderungen der ArbeitgeberInnen bzw. den Arbeitsanforderungen geformt, führt dies zu negativeren Bewertungen des Arbeitszeitmodells. Aus diesem Grund müssen ArbeitnehmerInnen vor einseitig von ArbeitgeberInnen bestimmter Flexibilität der Arbeitszeiten geschützt werden.

Damit kommt diese Untersuchung zu wichtigen Ergebnissen, zugleich werden Grenzen der vorliegenden Analyse deutlich. Die Beschränkung auf ausgewählte Branchen erlaubt keine empirisch gefestigten Aussagen für die gesamte Gruppe der abhängig Beschäftigten, auch vertiefende Vergleiche von einzelnen Branchen und kleineren Gruppen von ArbeitnehmerInnen sind aufgrund der Beschränkungen der Stichprobe nicht möglich. Zusätzlich konnte im Rahmen dieser Untersuchung nur beschränkt auf Besonderheiten unterschiedlicher Arbeitszeitlagen und unterschiedlicher Dauer in wechselnden Intervallen eingegangen werden. Hier besteht weiterer Forschungsbedarf um Ansatzpunkte für flexible Arbeitszeiten (auch) im Interesse von ArbeitnehmerInnen empirisch belegen zu können.

\section{Literatur}

Astleithner, Franz und Jörg Flecker (2017). From the golden age to the gilded cage? Austrian trade unions, social partnership and the crisis. In: Lehndorff, Steffen, Heiner Dribbusch und Thorsten Schulten (Hg.). Rough waters - European trade unions in a time of crises. Brussels: ETUI Printshop, 173-195.

Backhaus, Klaus, Bernd Erichson, Wulff Plinke und Rolf Weiber (2016). Multivariate Analysemethoden. Berlin, Heidelberg: Springer.

Chung, Heejung und Kea Tijdens (2013). Working time flexibility components and working time regimes in Europe: using company-level data across 21 countries. The International Journal of Human Resource Management, 24(7), 1418-1434.

Diaz-Bone, Rainer (2017). Statistik für Soziologen. 3. erw. Aufl. Konstanz: utb GmbH.

Dörre, Klaus (2010). Landnahme und soziale Zeitregimes. In: Frey, Michael (Hg.). Perspektiven auf Arbeit und Geschlecht: Transformationen, Reflexionen, Interventionen, Arbeit und Leben im Umbruch, München区i. Mering: Rainer Hampp Verlag, 47-72.

Eichmann, Hubert und Bernhard Saupe (2014). Überblick über Arbeitsbedingungen in Österreich. Follow-up-Studie: Studie der Forschungs- und Beratungsstelle Arbeitswelt (FORBA) im Auftrag des Sozialministeriums. Wien: Verlag des ÖGB.

Flecker, Jörg (2017). Arbeit und Beschäftigung: Eine soziologische Einführung. Wien: UTB GmbH.

Flecker, Jörg und Carina Altreiter (2014). Warum eine Arbeitszeitverkürzung sinnvoll ist. WISO, 3), $15-28$.

Griesbacher, Martin und Eva-Maria Griesbacher (2016). Herausforderungen selbstbestimmt-flexibler Arbeitszeiten in der unselbständigen Beschäftigung. Wien: Spectro gemeinnützige Gesellschaft für wissenschaftliche Forschung $\mathrm{GmbH}$.

Herrmann, Christoph et al. (2015). Arbeitszeitflexibilisierung auf betrieblicher Ebene. Forschungsprojekt für das Bundesministerium für Arbeit, Soziales und Konsumentenschutz. Wien: Forba. 
Hildebrandt, Eckart (2004). Balance von Arbeit und Leben - Neue Zumutungen oder Chance für mehr Lebensqualität? Arbeit, 13(4), 339-353.

Jürgens, Kerstin (2005). Die neue Unvereinbarkeit? Familienleben und flexibilisierte Arbeitszeiten. In: Seifert, Hartmut (Hg.). Flexible Zeiten in der Arbeitswelt. Frankfurt am Main, New York: Campus Verlag, 169-190.

Lott, Yvonne (2014). Flexibilität und Autonomie in der Arbeitszeit: Gut für die Work-Life Balance. Analysen zum Zusammenhang von Arbeitszeitarrangements und Work-Life Balance in Europa. WSI Report, 18, 1-16.

Matta, Vanita Irene (2015). Führen selbstgesteuerte Arbeitszeiten zu einer Ausweitung der Arbeitsstunden? Zeitschrift für Soziologie, 44(4), 253-271.

Moldaschl, Manfred und Günter G. Voß (2003). Subjektivierung von Arbeit. Arbeit, Innovation und Nachhaltigkeit. 2. Aufl. München: Hampp.

Peters, Klaus und Dieter Sauer (2005). Indirekte Steuerung - eine neue Herrschaftsform. In: Wagner, Hilde (Hg.) „Rentier ich mich noch?": neue Steuerungskonzepte im Betrieb, Hamburg: VSAVerlag, 23-58.

Reiner, Sabine (2015). Kurze Vollzeit für alle als Leitbild? Ökologisches Wirtschaften - Fachzeitschrift, $30(4), 26-27$.

Saupe, Bernhard und Bettina Stadler (2016). Arbeitszeitflexibilisierung - die Perspektive der ArbeitnehmerInnen. Projektbericht, Wien, FORBA.

Schönauer, Annika, Franz Astleithner, Matthias Nocker, Ingrid Mairhuber und Thomas Mittelberger (2016). Über- und Mehrstunden in Österreich: Entstehung und Verbreitung; Umgang im internationalen Vergleich, Wien: FORBA.

Seifert, Hartmut (2005). Flexible Zeiten in der Arbeitswelt. 1. Aufl. Frankfurt am Main: Campus Verlag.

Skinner, Natalie und Barbara Pocock (2011). Flexibility and work-life interference in Australia. Journal of Industrial Relations, 53(1), 65-82.

Voß, Günter G. (1998). Die Entgrenzung von Arbeit und Arbeitskraft. Mitteilungen aus der Arbeitsmarkt-und Berufsforschung, 31(3), 473-487.

Wunder, Christoph, und Guido Heineck (2013). Working time preferences, hours mismatch and wellbeing of couples: Are there spillovers? Labour Economics, 24, 244-252. 